\title{
Intractable hiccups caused by esophageal diverticular candidiasis in an immunocompetent adult: a case report
}

This article was published in the following Dove Press journal:

International Medical Case Reports Journal

10 February 2017

Number of times this article has been viewed

\author{
Shinsuke Yahata ${ }^{1,2}$ \\ Tsuneaki Kenzaka' \\ Saeko Kushida ${ }^{3}$ \\ Hogara Nishisaki ${ }^{2}$ \\ Hozuka Akita ${ }^{2}$ \\ 'Division of Community Medicine and \\ Career Development, Kobe University \\ Graduate School of Medicine, \\ Kobe, ${ }^{2}$ Department of Internal \\ Medicine, Hyogo Prefectural Kaibara \\ Hospital, Tamba, ${ }^{3}$ Department of \\ Gastroenterological Oncology, Hyogo \\ Cancer Center, Akashi, Japan
}

Introduction: Various causes of intractable hiccups have been reported; however, to the best of our knowledge, there are no previous reports of either intractable hiccups due to esophageal candidiasis in an immunocompetent adult or improvement following antifungal therapy.

Case presentation: An 87-year-old man presented with intractable hiccups. Although the patient was immunocompetent, he used proton pump inhibitors. An esophagogastroduodenoscopy revealed several white deposits throughout the esophagus and extensive white deposits in the midesophageal diverticulum. A mucosal culture showed candidiasis, which was suspected to be the cause of the intractable hiccups. After oral fluconazole had been prescribed, the candidiasis resolved and the hiccups improved. Therefore, we concluded that esophageal diverticular candidiasis was the cause of his intractable hiccups.

Conclusion: Physicians should consider esophageal candidiasis as one of the differential diagnoses for intractable hiccups, even in immunocompetent adults.

Keywords: Intractable hiccups, candidiasis, esophageal diverticulum, immunocompetence, elderly, acid-suppression therapy

\section{Introduction}

Hiccups result from brief and intense inspiratory bursts in the diaphragm and inspiratory intercostal muscles, with reciprocal inhibition of the expiratory intercostal muscles. After diaphragmatic contraction, glottic closure occurs immediately, minimizing the ventilatory effect and generating the characteristic sound and sense of discomfort. ${ }^{1}$ Hiccups are usually transient, but some patients occasionally experience intractable hiccups. Although the causes vary, there are only a few case reports of intractable hiccups associated with esophageal candidiasis, all of which occurred in patients with AIDS. ${ }^{2}$ Here, we report an extremely rare case of intractable hiccups caused by esophageal candidiasis in an immunocompetent adult.

\section{Case presentation}

An 87-year-old man presented to our hospital with intractable hiccups. In the last 2 years, he had twice experienced hiccups that persisted for several days and improved with the following symptomatic treatment from his primary physician: esomeprazole, metoclopramide, dimethicone, and prokinetic Chinese herbal medicine (i.e., rikkunshito and daikenchuto). Although this was his third episode of hiccups, he had not experienced them in the previous year. The patient described repetitive waxing and waning hiccup cycles lasting 3 days, with 4-7 days of relief. He took a Chinese herbal
Correspondence: Shinsuke Yahata unity Medicine and ment, Kobe University Graduate School of Medicine, 2-1-5 Aratacho, Hyogoku Kobe, Hyogo 652 0032, Japan

Tel +81783826732

Fax +81783826283

Email m0109Isy@jichi.ac.jp 
medicine with antispasmodic qualities, shakuyakukanzoto, which he received from his primary physician, and the symptoms resolved at first. However, 3 months later, the hiccups lasted for an entire day and did not improve with symptomatic treatment; therefore, he was referred to our hospital. He did not exhibit dysphagia, chest pain, chest discomfort, nausea, or headache.

The patient reported frequent use of esomeprazole for hiccups and chronic use of amlodipine and bisoprolol for hypertension. He had never used corticosteroids or any other immunosuppressant. His temperature, other vital signs, and the findings from a physical examination of the chest and abdomen were normal. Furthermore, he did not show signs of nuchal rigidity or cranial nerve abnormalities. His laboratory parameters were normal, with no evidence of an immunocompromised status, such as diabetes mellitus or AIDS (Table 1). Cranial magnetic resonance imaging revealed chronic lacunar infarcts; cerebral tumor or ventricle enlargement was not observed. Chest computed tomography showed a midesophageal diverticulum projecting rightward at the tracheal bifurcation (Figure 1). Lymph

Table I Laboratory data

\begin{tabular}{|c|c|c|}
\hline Parameter & Recorded value & Standard value \\
\hline White blood cell count $\left(10^{9} / \mathrm{L}\right)$ & 6.52 & $4.00-7.50$ \\
\hline Neutrophil & 4.15 & \\
\hline Eosinophil & 0.10 & \\
\hline Monocyte & 0.32 & \\
\hline Lymphocyte & 1.95 & \\
\hline Red blood cell count $\left(10^{12} / \mathrm{L}\right)$ & 4.49 & $4.00-5.50$ \\
\hline Hemoglobin $(g / d L)$ & 14.0 & $11.3-15.2$ \\
\hline Platelet count $\left(10^{9} / \mathrm{L}\right)$ & 131 & $130-350$ \\
\hline C-reactive protein $(\mathrm{mg} / \mathrm{dL})$ & 0.08 & $\leq 0.14$ \\
\hline Total protein (g/dL) & 7.8 & $6.9-8.4$ \\
\hline Albumin (g/dL) & 3.7 & $3.9-5.1$ \\
\hline Total bilirubin $(\mathrm{mg} / \mathrm{dL})$ & I.I & $0.4-1.5$ \\
\hline Aspartate aminotransferase (U/L) & 21 & $11-30$ \\
\hline Alanine aminotransferase (U/L) & 17 & $4-30$ \\
\hline Lactate dehydrogenase (U/L) & 202 & $109-216$ \\
\hline Alkaline phosphatase (U/L) & 199 & $107-330$ \\
\hline Creatine phosphokinase (U/L) & 116 & $45-290$ \\
\hline Blood nitrogen urea $(\mathrm{mg} / \mathrm{dL})$ & 17 & $8-20$ \\
\hline Creatinine $(\mathrm{mg} / \mathrm{dL})$ & 0.85 & $0.63-1.03$ \\
\hline Sodium (mEq/L) & 143 & $136-148$ \\
\hline Potassium (mEq/L) & 3.9 & $3.6-5.0$ \\
\hline Chloride $(\mathrm{mEq} / \mathrm{L})$ & 106 & $98-108$ \\
\hline Calcium (mg/dL) & 8.9 & $8.5-11.0$ \\
\hline Phosphorus (mg/dL) & 2.5 & $2.5-4.5$ \\
\hline Magnesium (mg/dL) & 2.46 & $1.8-2.5$ \\
\hline Glucose (mg/dL) & 114 & $70-109$ \\
\hline Glycohemoglobin (\%) & 5.7 & $4.7-6.2$ \\
\hline HIV-I/-2 antigen and antibody & Negative & \\
\hline Cutoff index & 0.3 & \\
\hline
\end{tabular}

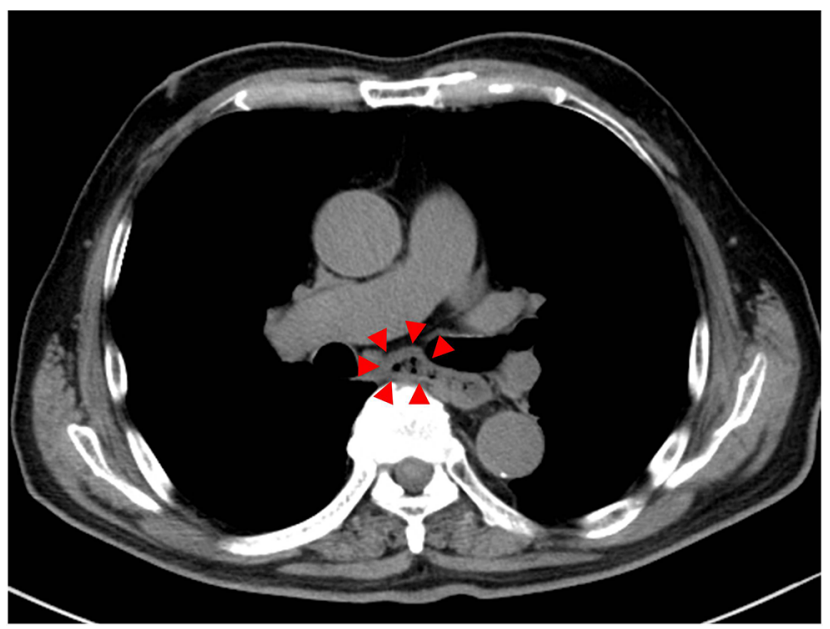

Figure I Midesophageal diverticulum on imaging. Chest computed tomography showing a midesophageal diverticulum (red arrows) projecting rightward at the tracheal bifurcation.

node swelling and other thoracoabdominal abnormalities were not observed.

An esophagogastroduodenoscopy (EGD) revealed several white deposits throughout the esophagus. The midesophageal diverticulum was localized on the right wall approximately 30 $\mathrm{cm}$ distal to the incisors (Figure 2). White residue was observed at the diverticulum margin and was flushed out easily. In the diverticulum, extensive white deposits were observed.

The deposits in the diverticulum were biopsied. Histopathological examination (hematoxylin and eosin staining) showed significant inflammatory cell infiltration and acantholytic finely fragmented squamous epithelial cells (Figure 3A), Grocott staining showed yeast and fungal filaments (Figure 3B), and mucosal culture revealed Candida albicans. Esophageal candidiasis was diagnosed. The patient was prescribed $200 \mathrm{mg}$ fluconazole once daily for 14 days, and the hiccups decreased markedly in frequency. When hiccups occurred, they stopped spontaneously within approximately 1 hour. After 2 weeks of treatment, the residue and white deposits were absent from the entire esophagus and diverticulum in the EGD views. The patient was monitored for 6 months and no recurrence was noted.

Written informed consent was obtained from the patient for publication of this case report and any accompanying images. The local ethics committee of Hyogo Prefectural Kaibara Hospital judged that the ethical review of this case was unnecessary.

\section{Discussion}

Virtually everyone experiences self-limiting, transient hiccups that last for several minutes or more. Hiccups are categorized according to their duration: "hiccup bouts" last 

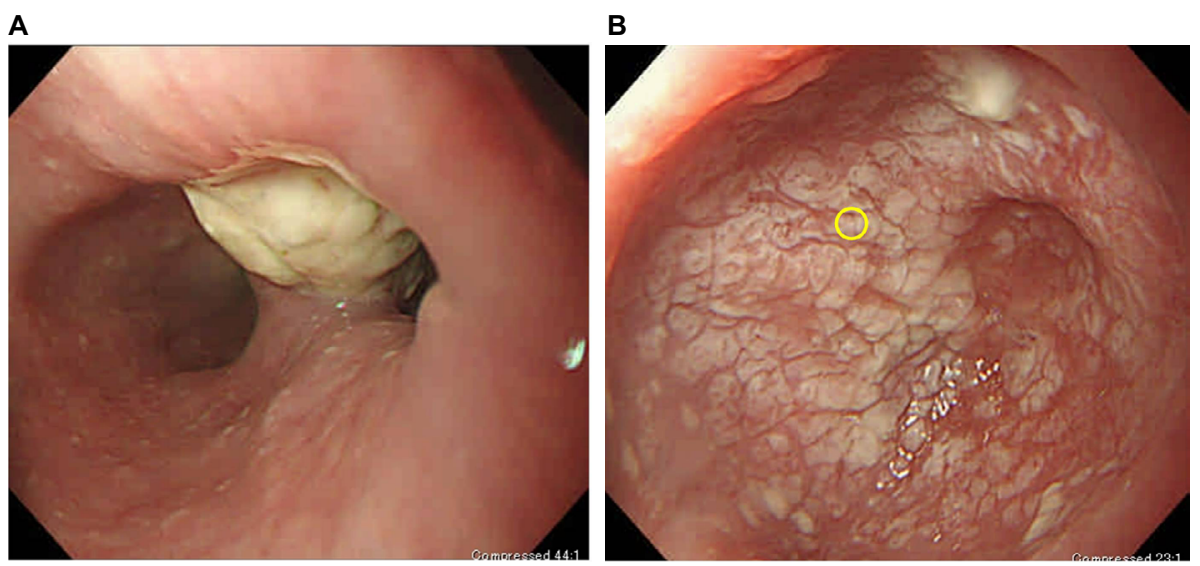

Figure 2 White deposits and residue in the esophagus. (A) Esophagogastroduodenoscopy revealing several white deposits throughout the esophagus. The midesophageal diverticulum was localized on the right wall approximately $30 \mathrm{~cm}$ distal to the incisors. White residue was observed at the diverticulum margin and was easily flushed out. (B) In the diverticulum, extensive white deposits were observed and biopsied (yellow circle).

A

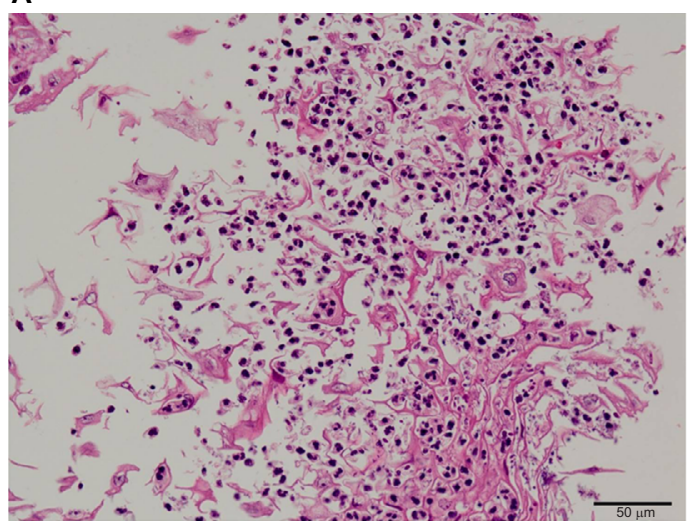

B

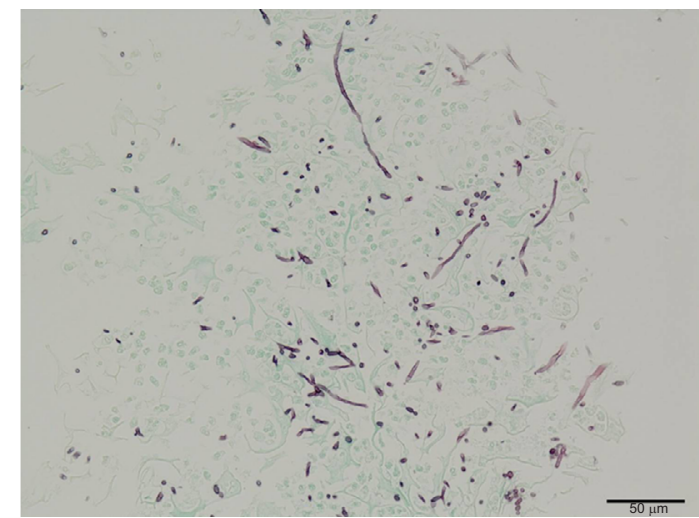

Figure 3 Histopathological findings. (A) Hematoxylin and eosin staining revealed significant inflammatory cell infiltration and acantholytic, finely fragmented squamous epithelial cells. (B) Grocott staining revealed yeast and fungal filaments. Scale bars represent $50 \mu \mathrm{m}$.

up to 48 hours, "persistent hiccups" last from 48 hours to 1 month, and "intractable hiccups" last longer than 1 month.

In our case, the patient had been experiencing intractable hiccups for 3 months. However, the intractable hiccups stopped after the esophageal candidiasis had been treated successfully with oral fluconazole, which is an antifungal agent that is recommended as a first-line treatment for candidiasis by the Japanese Society for Medical Mycology and the European Society of Clinical Microbiology and Infectious Diseases. ${ }^{4,5}$ Therefore, we concluded that his intractable hiccups were caused by esophageal candidiasis.

Although the mechanism underlying hiccups is unclear, it is thought that stimulation of the afferent portion of the hiccup reflex arc comprising the phrenic nerve, vagus nerve, and sympathetic chain is transmitted through central nervous system connections, such as the phrenic nerve nuclei, medullary reticular formation, and hypothalamus, and finally reaches the efferent component that is composed of the anterior scalene muscles, external intercostal muscles, glottis, and diaphragm. ${ }^{3}$ Transient hiccups are caused by gastric distension, sudden temperature change, alcohol ingestion, excessive smoking, and psychogenic factors. ${ }^{6}$ The causes of persistent or intractable hiccups vary appreciably and include irritation of the vagus nerve (e.g., pharyngitis, pneumonia, aortic aneurysm, and pancreatitis), central nervous system abnormalities (e.g., intracranial neoplasms, hydrocephalus, and meningitis), toxic-metabolic disease (e.g., uremia, diabetes mellitus, and electrolyte imbalance), diaphragmatic irritation (e.g., myocardial infarction, hepatosplenomegaly, and subphrenic abscess), drug intake (e.g., alpha methyldopa, short-acting barbiturates, and dexamethasone), infections (e.g. typhoid fever, influenza, and tuberculosis), general anesthesia, postoperative complications, and psychogenic or idiopathic variables. $^{6}$

Esophagitis can cause persistent or intractable hiccups because of irritation of the thoracic branches of the vagus nerve; however, to the best of our knowledge, there are only 
a few other case reports of intractable hiccups associated with esophageal candidiasis. ${ }^{2}$ Furthermore, the present report appears to be the first to describe a case of intractable hiccups caused by esophageal candidiasis in an immunocompetent adult. In our case, we suspect that inflammation of the esophagus stimulated the afferent portion of the hiccup reflex arc. The presence of inflammatory cell infiltration in the biopsied specimen suggests that inflammation of the esophagus might have contributed to the hiccups.

The symptoms of esophageal candidiasis are dysphagia, odynophagia, nausea, vomiting, retrosternal pain, and gastrointestinal bleeding, although some patients are asymptomatic. ${ }^{7-9}$ Because hiccups are atypical symptoms in esophageal candidiasis, they might be related to the esophageal diverticulum and the white residue, which was observed at the diverticulum margin. However, it is difficult to imagine that the esophageal diverticulum caused the intractable hiccups by itself because the diverticulum remained, even after the hiccups improved. Furthermore, because the white residue was flushed out easily, it is unlikely that it had irritated the vagus nerve and caused intractable hiccups of several months' duration.

Esophageal candidiasis usually occurs in immunocompromised hosts, although sometimes it is present in immunocompetent adults. Other risk factors for esophageal candidiasis are acid-suppression therapy (e.g., proton pump inhibitors or histamine-2 receptor blockers), elderly age, use of antibiotics, malignancy, diabetes mellitus, chronic renal failure, use of corticosteroids (systemic or inhalant), and use of another immunosuppressive agent. ${ }^{9-11}$ Our patient had some of these risk factors (i.e., elderly age and use of proton pump inhibitors). Furthermore, he had esophageal diverticulum. Although, we do not think that esophageal diverticulum itself caused the intractable hiccups, it may have become a hotbed of candidiasis. Perhaps consequently, he had esophageal candidiasis in spite of his immunocompetent status.

\section{Conclusion}

Clinicians should consider esophageal candidiasis as one of the differential diagnoses when treating a patient with intractable hiccups, especially if that patient is an elderly adult who is using acid-suppressing therapy or has existing esophageal diverticulum. Esophageal candidiasis should be considered even if the patient is immunocompetent.

\section{Disclosure}

The authors report no conflicts of interest in this work.

\section{References}

1. Howard RS. Persistent hiccups. BMJ. 1992;305(6864):1237-1238.

2. Albrecht H, Stellbrink HJ. Hiccups in people with AIDS. J Acquir Immune Defic Syndr.1994;7(7):735.

3. Kolodzik PW, Eilers MA. Hiccups (singultus): review and approach to management. Ann Emerg Med.1991;20(5):565-573.

4. Japanese Society of Mycology. [JSMM clinical practice guidelines for diagnosis and treatment of invasive candidiasis]. Med Mycol J. 2013;54(2):147-251. Japanese.

5. Ullmann AJ, Akova M, Herbrecht R, et al. ESCMID* guideline for the diagnosis and management of Candida diseases 2012: adults with haematological malignancies and after haematopoietic stem cell transplantation (HCT). Clin Microbiol Infect. 2012;18(Supp1 7):53-67.

6. Lewis JH. Hiccups: causes and cures. J Clin Gastroenterol. 1985;7(6): 539-552.

7. Eras P, Goldstein MJ, Sherlock P. Candida infection of the gastrointestinal tract. Medicine.1972;51(5):367-379.

8. López-Dupla M, Mora Sanz P, Pintado García V, et al. Clinical, endoscopic, immunologic, and therapeutic aspects of oropharyngeal and esophageal candidiasis in HIV-infected patients: a survey of 114 cases. Am J Gastroenterol. 1992;87(12):1771-1776.

9. Mimidis K, Papadopoulos V, Margaritis V, et al. Predisposing factors and clinical symptoms in HIV-negative patients with Candida oesophagitis: are they always present? Int J Clin Pract. 2005;59:210-213.

10. Kliemann DA, Pasqualotto AC, Falavigna M, Giaretta T, Severo LC. Candida esophagitis: species distribution and risk factors for infection. Rev Inst Med Trop Sao Paulo. 2008;50(5):261-263.

11. Kim KY, Jang JY, Kim JW, et al. Acid suppression therapy as a risk factor for Candida esophagitis. Dig Dis Sci. 2013;58(5):1282-1286.
International Medical Case Reports Journal

Publish your work in this journal

The International Medical Case Reports Journal is an international, peer-reviewed open-access journal publishing original case reports from all medical specialties. Previously unpublished medical posters are also accepted relating to any area of clinical or preclinical science. Submissions should not normally exceed 2,000 words or

\section{Dovepress}

4 published pages including figures, diagrams and references. The manuscript management system is completely online and includes a very quick and fair peer-review system, which is all easy to use. Visit http://www.dovepress.com/testimonials.php to read real quotes from published authors. 